Working Paper

Economics Series 10-27

October 2010
Departamento de Economía Universidad Carlos III de Madrid

Calle Madrid, 126

28903 Getafe (Spain)

Fax (34) 916249875

\title{
STRATEGY-PROOF ALLOCATION MECHANISMS FOR ECONOMIES WITH PUBLIC GOODS *
}

\author{
Diego Moreno $^{1}$ and María José Moscoso ${ }^{2}$
}

\begin{abstract}
We show that strategy-proof allocation mechanisms for economies with public goods are dictatorial -- i.e., they always select an allocation in their range that maximizes the welfare of the same single individual (the dictator). Further, strategy-proof and efficient allocation mechanisms are strongly dictatorial -- i.e., they select the dictator's preferred allocation on the entire feasible set. Thus, our results reveal the extent to which the conflict between individual incentives and other properties that may be deemed desirable (e.g., fairness, equal treatment, distributive justice) pervades resource allocation problems.
\end{abstract}

Keywords: Allocation mechanisms, public goods, strategy-proofness, dictatorship, efficiency.

${ }^{1}$ Moreno, Departamento de Economía, Universidad Carlos III de Madrid. dmoreno@eco.uc3m.es

${ }^{2}$ Moscoso, Departamento de Matemática Aplicada, E.U.I.T.I., Universidad Politécnica de Madrid. mariajose.moscoso@upm.es

\footnotetext{
*We gratefully acknowledge the comments and suggestions of the editor and referees. (In particular, we thank a referee who suggested a simple argument to deal with the case $n>2$ in the proof by induction of theorems 1 and 2.) We also thank Francisco Marhuenda and Carmelo Núñez for helpful discussion, and Carmen García-Miguel for composing Figure 2b. Financial support from the Spanish Ministry of Education, grants SEJ2007-67436 and Consolider-Ingenio 2010, and from the Comunidad de Madrid, grant Excelecon, is gratefully acknowledged.
} 


\section{Introduction}

Consider an economy in which there are a number of public goods (e.g., education, transportation, police, etc.) that can be produced from a single private good (e.g., money). It is well known that if each individual is to decide his contribution to the provision of public goods based only on his own preferences, then the resulting allocation will typically be suboptimal. Lindahl (1964) proposed a scheme for allocating public goods, which if implemented produces Pareto optimal outcomes. The viability of this scheme, however, was questioned by Samuelson (1954), who pointed out that it will generally not be compatible with individual incentives ("... it is in the selfish interest of each person to give false signals, to pretend to have less interest in a given collective consumption activity than he really has."). Hurwicz (1977) showed that the problem of incentive compatibility is not exclusive to public goods economies, but is generally present, even in pure private goods economies.

As decentralized market-like institutions such as voluntary contributions are not immune to manipulation by individuals, it seems natural to inquire into the properties of the alternative institutions (i.e., allocation mechanisms, or simply mechanisms) that are compatible with individual incentives. A mechanism is represented as a mapping that associates a feasible allocation with every profile of preferences reported by the individuals. Incentive compatible mechanisms are those for which an individual is always best off reporting his true preferences (i.e., for which truth-telling is a dominant strategy in the game form defined by the mechanism). Mechanisms having this property are referred to as strategy-proof.

This paper provides a characterization of the class of strategy-proof allocation mechanisms for economies with public goods. We consider domains of admissible preferences usually associated with economic environments, in which individuals are known to care only about their own allocation of goods, and preferences are assumed to have properties such as continuity, monotonicity, or convexity. We focus on $d i$ rect revelation mechanisms. The literature on implementation has considered more complex mechanisms in which agents' strategy spaces may include aspects other than their possible preferences. For the class of strategy-proof mechanisms, however, the revelation principle has established that the restriction to direct mechanisms poses no loss of generality.

Although the notion of incentive compatibility associated with strategy-proofness is very strong, it is the appropriate condition if one is to consider problems in which individuals have imperfect and asymmetric information about other individuals' pref- 
erences. Alternatively, one may introduce explicitly the information and beliefs of each individual about the other individuals' preferences or information, and model the situation as a game of incomplete information. In this context, incentive compatibility would require that truth-telling be a Bayesian-Nash equilibrium. Wilson (1987) has suggested that assuming that individuals' beliefs are common knowledge may be too strong. Furthermore, if truth-telling must be a Bayesian-Nash equilibrium for all possible profiles of individuals' beliefs, then an individual must be almost always best off reporting his true preferences - see Ledyard (1978). More recently, the literature has established that strategy-proofness is a necessary condition for robust implementation - see, e.g., Theorem 2 in Bergemann and Morris (2009).

In the social choice context, Gibbard (1973) and Satterthwaite (1975) have shown that if the domain of preferences is unrestricted, then strategy-proof decision mechanisms whose range contains at least three outcomes are dictatorial. (Dictatorial mechanisms select the outcome preferred by a single individual, the dictator, from the mechanism's range. Hence, dictatorial mechanisms resolve any conflict of interests that may arise in favor of the dictator.) Versions of the Gibbard-Satterthwaite Theorem have also been established for domains of preferences that satisfy properties commonly assumed in economic environments, such as continuity (Barberà and Peleg (1990)), continuity and convexity (Zhou (1991)), or continuity and monotonicity (Moreno (1999)). Nevertheless, the results established in these papers assume that individuals care about every dimension of the social outcome, and therefore have implications only for allocating pure public goods.

The present paper provides results showing that the Gibbard-Satterthwaite Theorem holds more generally. Specifically, for two domains of preferences that are standard in the economic literature, we show that strategy-proof mechanisms that must decide the allocation of public goods and also of other goods that affect the welfare of some, but not all, individuals (such as private goods, or externalities that are not fully public goods) are dictatorial. Furthermore, we show that efficient strategyproof mechanisms are strongly dictatorial - i.e., they select the dictator's preferred allocation on the entire feasible set. (A mechanism is efficient if for every profile of preferences it selects an allocation that is Pareto optimal with respect to that profile.) Consequently, our results establish a version of the Gibbard-Satterthwaite Theorem which applies to a broad class of economies, and reveal the extent to which the conflict between individual incentives and other properties that may be deemed desirable (e.g., fairness, equal treatment, distributive justice) pervades resource allocation problems. 
There is a great deal of literature studying mechanisms for public goods provision. For economies including one private good (on which individuals' preferences are assumed to be linear) and one or more public goods, Green and Laffont (1977) have characterized the class of strategy-proof and efficient mechanisms as Groves mechanisms; since mechanisms in this class are unbalanced, their characterization implies that strategy-proofness and efficiency are not compatible. For this domain of quasi-linear preferences, Hurwicz and Walker (1990) show that strategy-proof mechanisms will generally produce inefficient outcomes. For economies with private and public goods where individuals' preferences are represented by continuous, monotonic and concave utility functions, and each individual has access to a certain technology for producing public goods using private goods as inputs, Saijo (1991) shows that strategy-proofness and autarkic individual rationality are inconsistent.

Moreno and Walker (1991) study economies with public goods and other goods in which individuals' preferences are represented by strictly concave and quadratic utility functions, and show that strategy-proof mechanisms that satisfy conditional unanimity, and whose range projected on the set of possible public good bundles has dimension at least two, are dictatorial. In the same setting, Moreno (1994) considers the domain of preferences that are represented by continuous utility functions and shows that every strategy-proof and responsive mechanism whose range contains at least three outcomes that differ in the bundle of public goods is dictatorial. Schummer (1999) studies an economy with two agents who have linear preferences over one private good, and one or more public goods which are produced with a constant-returns to scale technology using the private good as input, and shows that strategy-proof and efficient mechanisms are dictatorial. Serizawa (1999) studies economies with one private good and one public good, and for the domain of preferences represented by utility functions that are continuous, strictly quasi-concave, and strictly monotonic, shows that strategy-proof, budget-balancing and symmetric mechanisms are equal cost sharing schemes.

In the present paper we study the strategy-proof mechanisms available for economies with public goods and other goods (e.g., private goods and/or externalities not fully public goods) for two alternative natural domains of preferences. We show (Theorem 1) that if preferences are represented by utility functions that are continuous, and increasing in the non-public dimensions, then any strategy-proof mechanism whose range contains at least three outcomes that differ in the bundle of public goods provided is dictatorial. Theorem 1 thus provides an extension of Barberà and Peleg's Theorem to economies with public goods and other goods. Note that, unlike Moreno 
(1994), we restrict the domain to contain only utility functions that are increasing in the non-public dimensions.

We also show (Theorem 2) that if preferences are represented by utility functions that are continuous, quasi-concave, and increasing in the non-public dimensions, then any strategy-proof mechanism whose range projected on the set of possible public good bundles has dimension at least two is dictatorial. Theorem 2 thus provides an extension of Zhou's Theorem to economies with public goods and other goods that are not public. Note that the assumption on the dimension of the mechanism's range in Theorem 2 requires the presence of at least two public goods, which rules out the economies studied by Serizawa (1999). Also note that unlike in Green and Laffont (1977) and Hurwicz and Walker (1990) the domain considered in Theorem 2 contains utility functions that are not quasi-linear. (Moreover, non-separable utility functions play a key role in the proof of Theorem 2.) Furthermore, unlike these papers, and Schummer (1999), Theorem 2 does not assume any particular structure of the feasible set, nor it is assumed that the non-public coordinates are private goods.

Moreover, in theorems 1 and 2 we do not impose efficiency as do Green and Laffont (1977), Hurwicz and Walker (1990) and Schummer (1999), nor do we impose individual rationally as does Saijo (1991), or conditional unanimity as do Moreno and Walker (1991), or responsiveness as does Moreno (1994). (Our Theorem 3, however, establishes a result analogous to that of Schummer (1999) for our general class of economies and the two domains of preferences we consider.)

For a general class of economies, Satterthwaite and Sonnenschein (1981) have established that strategy-proof, nonbossy and differentiable mechanisms are locally serially dictatorial. (A mechanism is nonbossy if no individual can change some other individual's outcome - by changing the utility function he reports - and maintain his own. Serially dictatorial mechanisms determine the allocation by selecting the preferred outcomes of a hierarchy of dictators.) The implications of the results of Satterthwaite and Sonnenschein (1981) are unclear: the conditions implying that a mechanism is globally dictatorial are very hard to check, and involve additional properties of the specific mechanism under consideration. Furthermore, the requirement that mechanisms be nonbossy rules out some potentially interesting mechanisms such as the "competitive mechanism," and raises the question as to whether there are bossy mechanisms that perform well. (Nonbossy mechanisms have been later studied by, among others, Serizawa (1996), and Deb and Ohseto (2002).) By contrast, our theorems 1 and 2 do not restrict attention to nonbossy mechanisms, and their scope and conclusions are unambiguous. 
Our results have no implications for economies with only pure private goods. Note, however, that strategy-proof and nondictatorial mechanisms do exist in this case. Consider, for example, an economy in which there are two private goods, $x$ and $y$, and three individuals. The economy is endowed with three units of $x$ and nothing of $y$. There is a constant returns to scale technology which yields one unit of $y$ for each unit of $x$ used as input. Let the mechanism $f$ assign to individuals 1 , 2 and 3, respectively, their most preferred consumption bundle whose cost in terms of $x$ does not exceed $1+m_{2}-m_{3}, 1+m_{3}-m_{1}$ and $1+m_{1}-m_{2}$ units of $x$, where $m_{i}$ is either zero if individual $i$ 's reported utility function is positive at $(0,0)$, and it is one otherwise. This mechanism is strategy-proof (no individual can influence his own outcome), and it is not dictatorial. Moreover, if individuals' preferences are monotonic, it produces Pareto optimal outcomes. (A similar example is discussed by Satterthwaite and Sonnenschein (1981).)

For a standard two person, two private goods pure exchange economy, Hurwicz (1972) has established that there are no incentive compatible mechanisms that always produce individually rational and Pareto optimal outcomes, and Zhou (1991) has shown that efficient mechanisms are dictatorial. For general pure exchange economies, Barberà and Jackson (1995) have characterized the class of strategy-proof anonymous and nonbossy mechanisms as those for which individuals trade according to fixed proportions. Other domains have been studied in the literature: Schummer (1997) investigates homothetic and strictly convex preferences; Serizawa (2002) and Serizawa and Weymark (2003) study classical homothetic and smooth preferences; Ju (2003) studies CES preferences; Nicoló (2004) studies Leontief preferences; and Hashimoto (2008) studies Cobb-Douglas preferences.

The extensive literature on strategy-proofness has been recently surveyed by Barberà (2010).

\section{$2 \quad$ The Model}

The set of individuals is $N=\{1, \ldots, n\}$. Individual $i$ 's consumption set is a subset of a finite dimensional Euclidean space, $X \times Y_{i} \subset \mathbb{R}_{+}^{m} \times \mathbb{R}_{+}^{l_{i}}$. The set of feasible allocations is $Z \subset X \times \prod_{i=1}^{n} Y_{i}$. Thus, the coordinate members of $X$ are the public goods to be provided, while the other coordinates are private goods or externalities which are not fully public goods. This representation includes allocation problems associated to pure public goods economies (for which the sets $Y_{i}$ are singletons), pure private goods economies (for which the set $X$ is a singleton), and mixed economies (i.e., economies 
in which there are public goods as well as private goods and/or other externalities).

The preferences of individual $i \in N$ are represented by utility functions, i.e., by real-valued functions on $X \times Y_{i}$. Since individuals utility functions might be known to have certain properties (e.g., to be continuous, or to be increasing in certain dimensions), for each $i \in N$ we denote by $U_{i}$ the set of individual $i$ 's a priori admissible utility functions. The set of admissible utility profiles is therefore $U=\prod_{i=1}^{n} U_{i}$. Utility profiles are denoted by $u=\left(u_{1}, \ldots, u_{n}\right)$. For $u \in U$ and $S \subset N$, we write $u_{-S}$ for the profile obtained from $u$ by deleting the utility functions of the members of $S$.

A mechanism is a mapping $f: U \rightarrow Z$. Given a mechanism $f$, we denote its range by $Z^{f}$, and for $u \in U$ we write $f_{x}(u) \in X$ the bundle of public goods provided, $f_{i}(u) \in$ $X \times Y_{i}$ for the bundle of goods received by individual $i$, and $f_{-i}(u) \in X \times \prod_{j \in N \backslash\{i\}} Y_{i}$ for the bundle of goods received by individuals other than $i$.

A mechanism $f$ is manipulable by individual $i$ at $u \in U$ if there is $\tilde{u}_{i} \in U_{i}$ such that $u_{i}\left(f_{i}\left(u_{-i}, \tilde{u}_{i}\right)\right)>u_{i}\left(f_{i}(u)\right)$. A mechanism is strategy-proof if it is not manipulable by any $i \in N$ at any $u \in U$. Strategy-proof mechanisms are those for which an individual is always best off reporting a utility function representing his true preferences (i.e., for which no individual can ever improve by reporting false preferences). Hence strategy-proofness guarantees that truth-telling is an equilibrium whatever individuals' preferences are.

For every set $A \subset X \times \prod_{i=1}^{n} Y_{i}$, we write $A_{X}$ and $A_{Y_{i}}$ for the projection of $A$ into $X$ and $Y_{i}$, respectively. We also use the (somewhat inconsistent but compact) notation $A_{i}$ and $A_{-i}$ to refer to the projection of $A$ into $X \times Y_{i}$ and $X \times \prod_{j \in N \backslash\{i\}} Y_{j}$, respectively. Also for a $A \subset \mathbb{R}^{p}$ we denote by $\bar{A}$ its closure, by $\# A$ its cardinality, and by $\operatorname{dim} A$ its dimension. (The dimension of a set is the dimension of the smallest affine subspace that contains the set).

A mechanism is dictatorial if there is an individual $i \in N$ such that for each $u \in U, f_{i}(u)$ maximizes $u_{i}$ on $Z_{i}^{f}$; individual $i$ is then referred to as a dictator for $f$. An mechanism is strongly dictatorial if there is an individual $i \in N$ such that for each $u \in U, f_{i}(u)$ maximizes $u_{i}$ on $Z_{i}$.

It should be noticed that the definition of dictatorial mechanisms given here is weaker than the one usually encountered in the social choice framework. Here the dictator preferences need only determine the dictator's consumption bundle. Of course, any conflict of interests between the dictator and other individuals is always settled in favor of the dictator. If there are public goods whose provision has to be decided, for example, then the decision will be made aiming to maximize the dictator's welfare. Thus, for economies with only public goods, this notion is the usual one. 


\section{The results}

Barberà and Peleg (1990), and Zhou (1991) have established that in pure public good economies, strategy-proof mechanisms are dictatorial. In our framework these theorems can be formulated as follows:

Theorem (Barberà and Peleg (1990)). Assume that $Y_{1}, \ldots, Y_{n}$ are singletons, and each $U_{i}$ contains all the continuous functions on $X \times Y_{i}$. Then every strategy-proof mechanism $f: U \longrightarrow Z$ such that $\# Z_{X}^{f} \geq 3$ is dictatorial.

Theorem (Zhou (1991)). Assume that $X$ is a convex set, $Y_{1}, \ldots, Y_{n}$ are singletons, and each $U_{i}$ contains all the continuous quasi-concave functions on $X \times Y_{i}$. Then every strategy-proof mechanism $f: U \longrightarrow Z$ such that $\operatorname{dim} Z_{X}^{f} \geq 2$ is dictatorial.

When $\# Z_{X}^{f}=2$ there are strategy-proof and nondictatorial mechanisms (e.g., majority voting). The condition on the dimension of $Z_{X}^{f}$ in Zhou's Theorem effectively requires that there be at least two public goods. When there is a single public good and individuals' preferences are represented by quasi-concave utility functions, then the class of strategy-proof mechanisms has been characterized by Moulin (1980) as median voter type mechanisms; hence, strategy-proof and nondictatorial mechanisms do exist in this case.

For the general class of allocation problems considered here, there may be certain natural restrictions on individuals' admissible utility functions. For example, if the non-public dimensions of an allocation problem are private goods that are always desirable or freely disposable, then admissible utility functions must be increasing in those dimensions. For each $i \in N$, a utility function $u_{i}$ is said to be $y_{i}$-increasing if for all $y_{i}, y_{i}^{\prime} \in Y_{i}$ with $y_{i}>y_{i}^{\prime}$, one has $u_{i}\left(x, y_{i}\right) \geq u_{i}\left(x, y_{i}^{\prime}\right)$. (For $a, b \in \mathbb{R}^{p}, a \geq b$ iff $a_{i} \geq b_{i}$ for all $i=1, \ldots, n$, and $a>b$ iff $a \geq b$ and $a \neq b$.)

Theorems 1 and 2 below generalize the above results to all allocation problems involving public goods; i.e., they apply to strategy-proof mechanisms for mixed economies (i.e., for economies in which there are private goods and/or other externalities, as well as public goods).

Theorem 1 establishes an analog to Barberà and Peleg's Theorem. Note that the sets of admissible utility functions are required to contain only utility functions that are increasing in the non-public dimensions.

Theorem 1. Assume that each $U_{i}$ contains all the continuous $y_{i}$-increasing functions on $X \times Y_{i}$. Then every strategy-proof mechanism $f: U \longrightarrow Z$ such that $\# Z_{X}^{f} \geq 3$ is dictatorial. 
Theorem 1 contains Barberà and Peleg's Theorem as a particular case. (However, Barberà and Peleg's Theorem plays a fundamental role in its proof.) It should be noticed that Theorem 1 does not require that the non-public dimensions be private goods; i.e., it allows the possibility for these dimensions to be goods of any nature: private goods, public goods or other externalities. Theorem 1 also contains Theorem 2 in Moreno (1994).

Theorem 2 below establishes an analog to Zhou's Theorem. Note that, as in Theorem 1, the set of admissible utility functions is required to contain only utility functions that are increasing in the non-public dimensions.

Theorem 2. Assume that $X, Y_{1}, \ldots, Y_{n}$ are convex sets, and each $U_{i}$ contains all the continuous, quasi-concave and $y_{i}$-increasing functions on $X \times Y_{i}$. Then every strategyproof mechanism $f: U \longrightarrow Z$ such that $\operatorname{dim} Z_{X}^{f} \geq 2$ is dictatorial.

Theorem 2 contains Zhou's Theorem as a particular case. (However, Zhou's Theorem plays a key role in its proof.) Theorem 2 also contains Moreno and Walker's (1991) Theorem. Note that economies with one public good and one private good are outside the scope of Theorem 2 as well, but strategy-proof and nondictatorial mechanisms do exist in this case - see Serizawa (1999).

The following remarks are helpful to make the scope of theorems 1 and 2 precise.

Remark 1. The condition on $Z_{X}^{f}$ in Theorem 1 (Theorem 2) effectively requires that at least one (two) public good(s) be provided.

Thus, theorems 1 and 2 have no implications for economies where there are no public goods. In particular, they have no implications for pure private good economies (i.e., for economies where $X$ is a singleton). As the example in the introduction shows, however, strategy-proof and nondictatorial mechanisms do exist for pure private good economies - see Barberà and Jackson (1995) for a characterization of the class of strategy-proof anonymous and nonbossy mechanisms.

Remark 2. The admissibility of utility functions that are non-separable (i.e., that are not of the form $\left.u_{i}\left(x, y_{i}\right)=v(x)+w\left(y_{i}\right)\right)$, and non-increasing in the public dimensions is essential in theorems 1 and 2.

Non-separable utility functions play a key role in the proof of theorems 1 and $2-$ see the proof on Lemma 3.2 in Section 4, and appendices A, B and C. Indeed, when only separable utility functions are admissible, there are strategy-proof and nondictatorial mechanisms - e.g., Groves type mechanisms; see Green and Laffont (1977). 
The admissibility of utility functions that are non-increasing in the public dimensions (i.e., admitting that individuals desire for public goods may be satiated) allows us to appeal to Barberà and Peleg's and Zhou's theorems in the proofs of Theorem 1 and Theorem 2, respectively. (It seems possible to obtain a result analogous to Theorem 1 when utility functions are increasing in all dimensions appealing to Moreno's (1999) Theorem 1, although there are a number of difficulties to overcome.)

As noted earlier, the definition of dictatorial mechanisms in our framework is weaker than the one usually encountered in the social choice framework. Here the dictator preferences need only determine the dictator's consumption bundle, perhaps leaving some room to determine the other coordinates of the allocation on the basis of reasonable criteria. Also, the mechanism's range may restrict the extent to which the dictator's interests prevail. If we ask a strategy-proof mechanism to produce Pareto optimal outcomes, however, then the allocation must be selected so as to maximize the dictator's welfare on the entire feasible set - i.e., the dictator becomes a strong dictator. This result is established in Theorem 3 below.

A mechanism $f$ is said to be efficient if for each $u \in U, f(u)$ is Pareto optimal with respect to $u$; i.e., there is no $(x, y) \in Z$ such that $u_{i}\left(x, y_{i}\right) \geq u_{i}\left(f_{i}(u)\right)$ for all $i \in N$ and $u_{j}\left(x, y_{j}\right)>u_{j}\left(f_{j}(u)\right)$ for some $j \in N$.

Theorem 3. Assume that $U$ and $Z$ satisfy either (3.1) or (3.2):

(3.1) Each $U_{i}$ contains all the continuous $y_{i}$-increasing functions on $X \times Y_{i}$ and $\# Z_{X} \geq 3$.

(3.2) The sets $X, Y_{1}, \ldots, Y_{n}$ are convex, each $U_{i}$ contains all the continuous, quasiconcave and $y_{i}$-increasing functions on $X \times Y_{i}$, and $\operatorname{dim} Z_{X} \geq 2$.

Then every strategy-proof and efficient mechanism $f: U \longrightarrow Z$ is strongly dictatorial.

Note that the cardinality and dimensionality conditions on Theorem 3 are imposed on the feasible set rather than on the range of the mechanism. Thus, Theorem 3 applies to a broad class of economies. Moreover, the strength of the conclusion of Theorem 3 makes it clear that imposing efficiency, in addition to strategy-proofness, leaves no room for any other desirable property such as fairness, equal treatment, distributive justice and the like.

In summary, we provide versions of the Gibbard-Satterthwaite Theorem that apply to a large class of economies and to domains of preferences with the properties commonly assumed in the literature. The persistence of the conclusion that mechanisms must either be manipulable or dictatorial reveals the impossibility of reconciling individuals' interests while maintaining other desirable properties. 


\section{The Proofs}

Given a set of admissible utility profiles $U$, denote by $U^{x}:=\prod_{1}^{n} U_{i}^{x}$ the set of profiles whose coordinate utility functions are constant on the non-public goods; i.e., for each $i \in N$ the set $U_{i}^{x}$ contains the utility functions $u_{i} \in U_{i}$ of the form $u_{i}\left(x, y_{i}\right)=v(x)$, where $v$ is a real-valued function on $X$.

The starting point in the proofs of theorems 1 and 2 is to show that the restriction to $U^{x}$ of a strategy-proof mechanism $f$ satisfying the assumptions of these theorems is dictatorial (Lemma 1). With this result in hand we establish some properties of the mechanisms obtained by fixing the utility function of a single individual other than the restricted dictator (i.e., the dictator of $f$ on $U^{x}$ ); specifically, we show that such mechanisms are strategy-proof, and that the projection of their range on the consumption set of the restricted dictator is virtually the same as that of the projection of the range of $f$ (lemmas 2 and 3 ). Then we prove by induction on the number of individuals that the dictator of $f$ on $U^{x}$ is in fact a dictator on the entire domain $U$. It turns out to be convenient to begin the induction argument with the case $n=2$. (Note, however, that for $n=1$ dictatorship and strategy-proofness are equivalent properties.)

Lemma 1. Let $f$ be a strategy-proof mechanism. Then $f_{x}\left(U^{x}\right)=Z_{X}^{f}$. Moreover, if $f$ satisfies the assumptions of either Theorem 1 or Theorem 2, then the restriction of $f$ to $U^{x}$ is dictatorial, and $Z_{X}^{f}$ is a closed set.

Proof. Let $f$ be a strategy-proof mechanism. We show that $f_{x}\left(U^{x}\right)=Z_{X}^{f}$. Obviously $f_{x}\left(U^{x}\right) \subset Z_{X}^{f}$. We show that $Z_{X}^{f} \subset f_{x}\left(U^{x}\right)$. Let $\bar{x} \in Z_{X}^{f}$, and let $\bar{u} \in U$ be such that $f_{x}(\bar{u})=\bar{x}$. For each $i \in N$, let $u_{i} \in U_{i}^{x}$ be given by $u_{i}\left(x, y_{i}\right)=-\|x-\bar{x}\|$. Since $f$ is strategy-proof, one has $f_{x}\left(u_{1}, \bar{u}_{-1}\right)=\bar{x}$ (otherwise $f$ is manipulable by individual 1 at $\bar{u}$. Similarly,

$$
f_{x}\left(u_{1}, \bar{u}_{-1}\right)=f_{x}\left(u_{1}, u_{2}, \bar{u}_{-\{1,2\}}\right)=\ldots=f_{x}\left(u_{1}, \ldots, u_{n}\right)=\bar{x} .
$$

Hence $\bar{x} \in f_{x}\left(U^{x}\right)$.

Assume now that $f$ satisfies the assumptions of either Theorem 1 or Theorem 2 . Let $\stackrel{\circ}{y} \in \prod_{1}^{n} Y_{i}$ be arbitrary, and for each $i \in N$, let $\stackrel{\circ}{U}_{i}$ denote the set of utility functions on $X \times\left\{\stackrel{\circ}{y}_{i}\right\}$ that are restrictions of functions in $U_{i}^{x}$. Note that for each $\stackrel{\circ}{u}_{i} \in \stackrel{\circ}{U}_{i}$, there is a unique $u_{i} \in U_{i}^{x}$ such that $\stackrel{\circ}{i}_{i}\left(x, \stackrel{\circ}{y}_{i}\right)=u_{i}\left(x, y_{i}\right)$ for each $\left(x, y_{i}\right) \in X \times Y_{i}$. Write $\stackrel{\circ}{u}_{i} \approx u_{i}$ when the functions $\stackrel{\circ}{u}_{i}$ and $u_{i}$ are related in this way.

Write $\stackrel{\circ}{U}=\prod_{i=1}^{n} \stackrel{\circ}{U}_{i}$, and $\stackrel{\circ}{Z}=X \times\{\stackrel{\circ}{y}\}$, and define the mechanism $g: \stackrel{\circ}{U} \longrightarrow \stackrel{\circ}{Z}$ by $g(\stackrel{\circ}{u})=\left(f_{x}(u), \stackrel{\circ}{y}\right)$, where $u \in U^{x}$ is such that for each $i \in N, \stackrel{\circ}{u}_{i} \approx u_{i}$. Clearly, 
$g$ is strategy-proof. Suppose not; let $j \in N, \stackrel{\circ}{\dot{u}} \in \stackrel{\circ}{U}$ and $\stackrel{\circ}{u}_{j}^{\prime} \in \stackrel{\circ}{U}_{j}$ be such that $\stackrel{\circ}{u}_{j}\left(g_{j}\left(\stackrel{\circ}{u}_{j}^{\prime}, \stackrel{\circ}{u}_{-j}\right)\right)>\stackrel{\circ}{u}_{j}\left(g_{j}(\stackrel{\circ}{u})\right)$. Also let $u=\left(u_{1}, \ldots, u_{n}\right) \in U^{x}$ and $u_{j}^{\prime} \in U_{j}^{x}$ be such that $\stackrel{\circ}{i}_{i} \approx u_{i}$ for each $i \in N$, and $\stackrel{u}{j}_{j}^{\prime} \approx u_{j}^{\prime}$. Then one has

$$
u_{j}\left(f_{j}\left(u_{j}^{\prime}, u_{-j}\right)\right)=u_{j}\left(g_{j}\left(\stackrel{\circ}{u}_{j}^{\prime}, \stackrel{\circ}{-j}_{-j}\right)\right)>u_{j}\left(g_{j}(\stackrel{\circ}{u})\right)=u_{j}\left(f_{j}(u)\right),
$$

and therefore $f$ is manipulable by individual $j$ at $u \in U^{x}$, which contradicts that $f$ is strategy-proof. Hence $g$ is strategy-proof, and therefore, as shown above, $\check{Z}^{g}=$ $Z_{X}^{f} \times\{\grave{y}\}$.

If the assumptions of Theorem 1 hold, then Barberà and Peleg's Theorem implies that $g$ is dictatorial. And if the assumptions of Theorem 2 hold, then Zhou's Theorem implies that $g$ is dictatorial. Therefore, in either case $g$ is dictatorial.

Without loss of generality assume that individual 1 is the dictator of $g$. We show that individual 1 is a dictator for $f$ on $U^{x}$. Let $u \in U^{x}$, and let $\stackrel{\circ}{u} \stackrel{\circ}{U}$ be such that $\stackrel{\circ}{u}_{i} \approx u_{i}$ for each $i \in N$. Since $g_{1}(\stackrel{\circ}{u})$ maximizes $\stackrel{\circ}{u}_{1}$ on $\stackrel{\circ}{Z}_{1}^{g}$ we have $\stackrel{\circ}{u}_{1}\left(g_{1}(\stackrel{\circ}{u})\right) \geq \stackrel{\circ}{u}_{1}\left(x, \stackrel{\circ}{y}_{1}\right)$ for all $\left(x, \stackrel{\circ}{y}_{1}\right) \in \stackrel{\circ}{Z}_{1}^{g}$. And since $\stackrel{\circ}{u}_{1}\left(g_{1}(\stackrel{\circ}{u})\right)=\stackrel{\circ}{u}_{1}\left(f_{x}(u), \stackrel{\circ}{y}_{1}\right)=u_{1}\left(f_{1}\left(u^{x}\right)\right)$, then

$$
u_{1}\left(f_{1}\left(u^{x}\right)\right) \geq u_{1}\left(x, \stackrel{\circ}{1}_{1}\right)=u_{1}\left(x, y_{1}\right)
$$

for all $\left(x, y_{1}\right) \in Z_{1}^{f}$. Hence $f$ is dictatorial on $U^{x}$.

Finally, we show that $Z_{X}^{f}$ is closed. Let $\bar{x}$ be a member of the closure of $Z_{X}^{f}$, and let $\left(u_{1}, \ldots, u_{n}\right) \in U^{x}$ be such that for $\left(x, y_{1}\right) \in X \times Y_{1}$, we have $u_{1}\left(x, y_{1}\right)=-\|x-\bar{x}\|$. Suppose that $f_{x}\left(u_{1}, \ldots, u_{n}\right)=\hat{x} \neq \bar{x}$. Since $\bar{x}$ is in the closure of $Z_{X}^{f}$ there is $\bar{x}^{\prime} \in Z_{X}^{f}$ sufficiently close to $\bar{x}$ that $-\left\|\bar{x}^{\prime}-\bar{x}\right\|>-\|\hat{x}-\bar{x}\|$. Since $Z_{X}^{f}=f_{x}\left(U^{x}\right)$, there is $\left(\bar{x}^{\prime}, y_{1}\right) \in f_{1}\left(U^{x}\right)$ such that

$$
u_{1}\left(\bar{x}^{\prime}, y_{1}\right)=-\left\|\bar{x}^{\prime}-\bar{x}\right\|>-\|\hat{x}-\bar{x}\|=u_{1}\left(f_{1}\left(u_{1}, \ldots, u_{n}\right)\right)
$$

which contradicts that individual 1 is a dictator for $f$ on $U^{x}$.

For our next lemma we need to introduce some additional notation. Let $f$ be a mechanism, and let $i \in N$. For each $u_{i} \in U_{i}$ let the mapping $f^{u_{i}}: \prod_{j \in N \backslash\{i\}} U_{j} \longrightarrow Z_{-i}$ be given for each $u_{-i} \in \prod_{j \in N \backslash\{i\}} U_{j}$ by $f^{u_{i}}\left(u_{-i}\right)=f_{-i}\left(u_{i}, u_{-i}\right)$. Note that each $f^{u_{i}}$ is a mechanism.

Lemma 2. Let $f$ be a strategy-proof mechanism satisfying the assumptions of either Theorem 1 or Theorem 2, and assume, w.l.o.g., that individual 1 is the dictator of $f$ on $U^{x}$ (Lemma 1). Then for all $i \in N \backslash\{1\}$ and $u_{i} \in U_{i}$ :

(2.1) $f^{u_{i}}$ is strategy-proof and $Z_{X}^{f^{u_{i}}}=Z_{X}^{f}$.

(2.2) If $f^{u_{i}}$ is dictatorial, then individual 1 is the dictator. 
Proof. Let $i \in N \backslash\{1\}$ and $u_{i} \in U_{i}$. We prove Lemma 2.1. We first show that $f^{u_{i}}$ is strategy-proof. Suppose by way of contradiction that $f^{u_{i}}$ is manipulable by $k \in N \backslash\{i\}$ at $u_{-i} \in \prod_{j \in N \backslash\{i\}} U_{i}$ via $\tilde{u}_{k} \in U_{k}$; then

$$
u_{k}\left(f_{k}\left(u_{i}, \tilde{u}_{k}, u_{-\{i, k\}}\right)\right)=u_{k}\left(f_{k}^{u_{i}}\left(\tilde{u}_{k}, u_{-\{i, k\}}\right)\right)>u_{k}\left(f_{k}^{u_{i}}\left(u_{-i}\right)\right)=u_{k}\left(f_{k}\left(u_{i}, u_{-i}\right),\right.
$$

and therefore $f$ is manipulable by individual $k$ at $\left(u_{i}, u_{-i}\right) \in U$, contradicting that $f$ is strategy-proof.

Next, we prove that $Z_{X}^{f^{u_{i}}}=Z_{X}^{f}$. Since $Z_{X}^{f^{u_{i}}} \subset Z_{X}^{f}$, we show that $Z_{X}^{f} \subset Z_{X}^{f^{u_{i}}}$. Let $\bar{x} \in Z_{X}^{f}$, and suppose that $\bar{x} \notin Z_{X}^{f^{u_{i}}}$. Let $\bar{u}_{1} \in U_{1}^{x}$ be given by $\bar{u}_{1}\left(x, y_{1}\right)=-\|x-\bar{x}\|$. Since $\bar{x} \notin Z_{X}^{f^{u_{i}}}$, then

$$
f_{x}^{u_{i}}\left(\bar{u}_{1}, u_{-\{1, i\}}\right)=\tilde{x}
$$

where $\tilde{x} \neq \bar{x}$. For $j \in N \backslash\{1\}$, let $\tilde{u}_{j} \in U_{j}^{x}$ be given by $\tilde{u}_{j}\left(x, y_{j}\right)=-\|x-\tilde{x}\|$. Since $f$ is strategy-proof one has

$$
f_{x}\left(\bar{u}_{1}, \tilde{u}_{i}, u_{-\{1, i\}}\right)=f_{x}\left(\bar{u}_{1}, \tilde{u}_{2}, \tilde{u}_{i}, u_{-\{1,2, i\}},\right)=\ldots=f_{x}\left(\bar{u}_{1}, \tilde{u}_{2}, \ldots, \tilde{u}_{n}\right)=\tilde{x} \neq \bar{x} .
$$

But note that $\left(\bar{u}_{1}, \tilde{u}_{2}, \ldots, \tilde{u}_{n}\right) \in U^{x}$, and therefore this contradicts that individual 1 is the dictator for the restriction of $f$ to $U^{x}$. Hence $Z_{X}^{f} \subset Z_{X}^{f^{u_{i}}}$.

We prove Lemma 2.2. Suppose by way of contradiction that some individual $k \in N \backslash\{1, i\}$ is the dictator of $f^{u_{i}}$. Let $\bar{x}, \tilde{x} \in Z_{X}^{f^{u_{i}}}=Z_{X}^{f}$, with $\bar{x} \neq \tilde{x}$, and let $\bar{u}_{1}$ and $\tilde{u}_{j}$ for $j \in N$ as defined in the proof of Lemma 2.1 above. Write $\tilde{u}=\left(\tilde{u}_{1}, \ldots, \tilde{u}_{n}\right)$. Since individual $k \in N \backslash\{1, i\}$ is the dictator of $f^{u_{i}}$, then we have

$$
\left.f_{x}^{u_{i}}\left(\bar{u}_{1}, \tilde{u}_{-\{1, i\}}\right)\right)=f_{x}\left(\bar{u}_{1}, u_{i}, \tilde{u}_{-\{1, i\}}\right)=\tilde{x} .
$$

And since $f$ is strategy-proof, then

$$
f_{x}\left(\bar{u}_{1}, \tilde{u}_{i}, \tilde{u}_{-\{1, i\}}\right)=\tilde{x} \neq \bar{x} .
$$

However, $\left(\bar{u}_{1}, \tilde{u}_{i}, \tilde{u}_{-\{1, i\}}\right) \in U^{x}$, which contradicts that individual 1 is the dictator for the restriction of $f$ to $U^{x}$.

We define a family of Leontief-type functions, $\left\{w_{\hat{y}_{1}}\right\}_{\hat{y}_{1} \in Y_{1} \backslash\{0\}}$, that will be useful in the rest of the proof. Let $\hat{y}_{1} \in Y_{1} \backslash\{0\}$; if $\hat{y}_{1}^{k}>0$ for all $k$, then the function $w_{\hat{y}_{1}}: Y_{1} \rightarrow[0,1]$ is given for $y_{1} \in Y_{1}$ by

$$
w_{\hat{y}_{1}}\left(y_{1}\right)=\frac{1}{\alpha_{1} \hat{y}_{1}^{1}} \min \left\{\alpha_{1} y_{1}^{1}, \ldots, \alpha_{l_{1}} y_{1}^{l_{1}} ; \alpha_{1} \hat{y}_{1}^{1}\right\}
$$

where $\alpha=\left(\alpha_{1}, \ldots, \alpha_{l_{1}}\right) \in \mathbb{R}_{+}^{l_{1}}$ satisfies $\alpha_{1} \hat{y}_{1}^{1}=\alpha_{2} \hat{y}_{1}^{2}=\ldots=\alpha_{l_{1}} \hat{y}_{1}^{l_{1}}$. If $\hat{y}_{1}^{k}=0$ for some $k$, then the term $\alpha_{k} \hat{y}_{1}^{k}$ is suppressed from the formula above and from the set 
equations that the vector $\alpha$ must satisfy, and if $\hat{y}_{1}^{1}=0$, then the term $\alpha_{1} \hat{y}_{1}^{1}$ in the formula is replaced by the term $\alpha_{k} \hat{y}_{1}^{k}$ corresponding to the first positive coordinate of $\hat{y}_{1}$. Note that each $w_{\hat{y}_{1}}$ is an increasing, continuous, and concave function, and satisfies $w_{\hat{y}_{1}}\left(y_{1}\right)=1$ for $y_{1} \geq \hat{y}_{1}$.

For $\hat{x} \in X$ and $A \subset X$, denote by $d(\hat{x}, A)=\inf _{x \in A}\|\hat{x}-x\|$ the distance from $\hat{x}$ to $A$. Also, given a mechanism $f$ and a point $\bar{x} \in Z_{X}^{f}$, write

$$
Z_{Y_{1}}^{f}(\bar{x}):=\left\{y_{1} \in Y_{1} \mid\left(\bar{x}, y_{1}\right) \in Z_{1}^{f}\right\}
$$

Lemma 3. Let $f$ be a strategy-proof mechanism satisfying the assumptions of either Theorem 1 or Theorem 2. Assume, w.l.o.g., that individual 1 is the dictator of $f$ on $U^{x}$ (Lemma 1), and suppose that $n=2$. Then, for all $u_{2} \in U_{2}$ and all $\bar{x} \in Z_{X}^{f}$ :

(3.1) $Z_{Y_{1}}^{f_{2}}(\bar{x})$ is non-empty and bounded, and contains every $\bar{y}_{1} \in \overline{Z_{Y_{1}}^{f^{u_{2}}}(\bar{x})}$ such that no $y_{1} \in Z_{Y_{1}}^{f_{2}}(\bar{x})$ satisfies $y_{1}>\bar{y}_{1}$. Moreover, for all $\hat{y}_{1} \in Y_{1} \backslash\{0\}$, w $w_{\hat{y}_{1}}$ has a maximum on $Z_{Y_{1}}^{f^{u_{2}}}(\bar{x})$.

(3.2) For each $y_{1} \in Z_{Y_{1}}^{f}(\bar{x})$ there is $y_{1}^{\prime} \in Z_{Y_{1}}^{f_{2}}(\bar{x})$ such that $y_{1}^{\prime} \geq y_{1}$.

Proof. Let $u_{2} \in U_{2}$ and $\bar{x} \in Z_{X}^{f}$.

We establish (3.1). Since $Z_{X}^{f^{u_{2}}}=Z_{X}^{f}$ by Lemma 2.1, then $Z_{Y_{1}}^{f^{u_{2}}}(\bar{x})$ is non-empty. We show that $Z_{Y_{1}}^{f_{2}}(\bar{x})$ is bounded. Let $u_{1} \in U_{1}$ be given for all $\left(x, y_{1}\right) \in X \times Y_{1}$ by

$$
u_{1}\left(x, y_{1}\right)=-\|x-\bar{x}\|+\sum_{j=1}^{l_{1}} y_{1}^{j} .
$$

If $Z_{Y_{1}}^{f_{2}}(\bar{x})$ is unbounded, then $u_{1}$ does not have a maximizer on $Z_{1}^{f^{u_{2}}}$, which contradicts that $f^{u_{2}}$ is strategy-proof (Lemma 2.1), i.e., that $f_{1}^{u_{2}}\left(u_{1}\right)$ maximizes $u_{1}$ on $Z_{1}^{f^{u_{2}}}$. Hence $Z_{Y_{1}}^{f_{2}}(\bar{x})$ is bounded.

Let $\bar{y}_{1} \in \overline{Z_{Y_{1}}^{f_{2}}(\bar{x})}$ be such that there is no $y_{1}^{\prime} \in Z_{Y_{1}}^{f_{2}}(\bar{x})$ with $y_{1}^{\prime}>\bar{y}_{1}$. We show that $\bar{y}_{1} \in Z_{Y_{1}}^{f^{u_{2}}}(\bar{x})$. Let $\bar{u}_{1}$ be given for all $\left(x, y_{1}\right) \in X \times Y_{1}$ by $\bar{u}_{1}\left(x, y_{1}\right)=-\|x-\bar{x}\|+w_{\bar{y}_{1}}\left(y_{1}\right)$. We have

$$
\bar{u}_{1}\left(\bar{x}, \bar{y}_{1}\right)-\bar{u}_{1}\left(x, y_{1}\right)=1-w_{\bar{y}_{1}}\left(y_{1}\right)+\|x-\bar{x}\|
$$

If $x \neq \bar{x}$, then

$$
\bar{u}_{1}\left(\bar{x}, \bar{y}_{1}\right)-\bar{u}_{1}\left(x, y_{1}\right) \geq\|x-\bar{x}\|>0 .
$$

If $y_{1} \neq \bar{y}_{1}$ and $\left(x, y_{1}\right) \in Z_{1}^{f^{u_{2}}}$, since no $y_{1}^{\prime} \in Z_{Y_{1}}^{f_{2}}(\bar{x})$ satisfies $y_{1}^{\prime}>\bar{y}_{1}$, then we have

$$
\bar{u}_{1}\left(\bar{x}, \bar{y}_{1}\right)-\bar{u}_{1}\left(x, y_{1}\right) \geq 1-w_{\bar{y}_{1}}\left(y_{1}\right)>0 \text {. }
$$


Assume that $f_{1}^{u_{2}}\left(\bar{u}_{1}\right) \neq\left(\bar{x}, \bar{y}_{1}\right)$; then $\bar{u}_{1}\left(\bar{x}, \bar{y}_{1}\right)-\bar{u}_{1}\left(f_{1}^{u_{2}}\left(\bar{u}_{1}\right)\right)>0$. Since $\bar{y}_{1}$ is in the closure of $Z_{Y_{1}}^{f^{u_{2}}}(\bar{x})$, then we can find $\tilde{y}_{1} \in Z_{Y_{1}}^{f^{u_{2}}}(\bar{x})$ sufficiently close to $\bar{y}_{1}$ that

$$
\bar{u}_{1}\left(\bar{x}, \bar{y}_{1}\right)-\bar{u}_{1}\left(\bar{x}, \tilde{y}_{1}\right)=1-w_{\bar{y}_{1}}\left(\tilde{y}_{1}\right)<\bar{u}_{1}\left(\bar{x}, \bar{y}_{1}\right)-\bar{u}_{1}\left(f_{1}^{u_{2}}\left(\bar{u}_{1}\right)\right) ;
$$

i.e.,

$$
\bar{u}_{1}\left(\bar{x}, \tilde{y}_{1}\right)>\bar{u}_{1}\left(f_{1}^{u_{2}}\left(\bar{u}_{1}\right)\right)
$$

which contradicts that $f^{u_{2}}$ is strategy-proof (Lemma 2.1). Hence $f_{1}^{u_{2}}\left(\bar{u}_{1}\right)=\left(\bar{x}, \bar{y}_{1}\right)$, and therefore $\bar{y}_{1} \in Z_{Y_{1}}^{f^{u_{2}}}(\bar{x})$.

Finally, let $\hat{y}_{1} \in Y_{1} \backslash\{0\}$. Since $w_{\hat{y}_{1}}$ is increasing, it has a maximizer $y_{1}$ on $\overline{Z_{Y_{1}}^{f^{u_{2}}}(\bar{x})}$ such that there is no $y_{1}^{\prime} \in Z_{Y_{1}}^{f^{u_{2}}}(\bar{x})$ with $y_{1}^{\prime}>y_{1}$. Then $y_{1} \in Z_{Y_{1}}^{f_{2}}(\bar{x})$ as shown above, and therefore $w_{\hat{y}_{1}}\left(y_{1}\right)$ is the maximum value of $w_{\hat{y}_{1}}$ on $Z_{Y_{1}}^{f^{u_{2}}}(\bar{x})$.

We prove (3.2). Suppose by way of contradiction that there is $\bar{y}_{1} \in Z_{Y_{1}}^{f}(\bar{x})$ such that no $y_{1} \in Z_{Y_{1}}^{f_{2}}(\bar{x})$ satisfies $y_{1} \geq \bar{y}_{1}$. Let $\hat{u}_{1} \in U_{1}$ be a continuous, concave and $y_{1}$-increasing utility function satisfying

(a) $\hat{u}_{1}\left(\bar{x}, \bar{y}_{1}\right)>\hat{u}_{1}\left(x, y_{1}\right)$ for all $\left(x, y_{1}\right) \in Z_{1}^{f} \backslash\left\{\left(\bar{x}, y_{1}^{\prime}\right) \in Z_{1} \mid y_{1}^{\prime} \geq \bar{y}_{1}\right\}$, and such that

(b) every maximizer $\left(x, y_{1}\right)$ of $\hat{u}_{1}$ on $Z_{1}^{f^{u_{2}}}$ satisfies $x \neq \bar{x}$.

(A utility function with these properties is constructed in Appendix A.) Since $f^{u_{2}}$ is strategy-proof (Lemma 2.1), then $f^{u_{2}}\left(\hat{u}_{1}\right)$ maximizes $\hat{u}_{1}$ on $Z_{1}^{f^{u_{2}}}$, and therefore $f_{x}^{u_{2}}\left(\hat{u}_{1}\right) \neq \bar{x}$. Write $f_{x}^{u_{2}}\left(\hat{u}_{1}\right)=f_{x}\left(\hat{u}_{1}, u_{2}\right)=\overline{\bar{x}}$.

For each $\hat{u}_{2} \in U_{2}$ such that $\hat{u}_{2}\left(x, y_{2}\right)=\hat{v}(x)$, where $\hat{v}$ is an arbitrary continuous function uniquely maximized on $Z_{X}^{f}$ at $\overline{\bar{x}}$, we have $f_{x}\left(\hat{u}_{1}, \hat{u}_{2}\right)=\overline{\bar{x}}$, for otherwise $f$ is manipulable at $\left(\hat{u}_{1}, \hat{u}_{2}\right)$. Further, there is no $\left(\bar{x}, y_{1}\right) \in Z_{1}^{f^{\hat{u}_{2}}}$ with $y_{1} \geq \bar{y}_{1}$, for otherwise $f^{\hat{u}_{2}}\left(\hat{u}_{1}\right)$ does not maximize $\hat{u}_{1}$ on $Z_{1}^{f^{\hat{u}_{2}}}$, which contradicts that $f^{\hat{u}_{2}}$ is strategy-proof.

We complete the proof of Lemma 3.2 under the assumptions of Theorem 1. By assumption, there is $\overline{\bar{x}} \in Z_{X}^{f} \backslash\{\bar{x}, \overline{\bar{x}}\}$. Let $\hat{u}_{2} \in U_{2}$ be such that $\hat{u}_{2}\left(x, y_{2}\right)=\hat{v}(x)$, where $\hat{v}$ is a continuous function uniquely maximized on $Z_{X}^{f}$ at $\overline{\bar{x}}$, and satisfying $\hat{v}(\bar{x})>\hat{v}(\overline{\bar{x}})$ - see the proof of Barberà and Peleg (1990)'s Lemma 5.6 for an example of a utility function with these properties. Then $f_{x}\left(\hat{u}_{1}, \hat{u}_{2}\right)=\overline{\bar{x}}$. Let $\tilde{u}_{1} \in U_{1}$ be a continuous utility function satisfying (a) above, and such that

(c.1) every maximizer $\left(x, y_{1}\right)$ of $\tilde{u}_{1}$ on $Z_{1}^{f^{\hat{u}_{2}}}$ satisfies $x=\overline{\bar{x}}$.

(A utility function with these properties is constructed in Appendix B.) Since $f^{\hat{u}_{2}}$ is strategy-proof, then $f_{x}^{\hat{u}_{2}}\left(\tilde{u}_{1}\right)=\overline{\bar{x}}$. Since $\left(\bar{x}, \bar{y}_{1}\right) \in Z_{1}^{f}$, then there is $\bar{u}_{2} \in U_{2}$ such that $\left(\bar{x}, \bar{y}_{1}\right) \in Z_{1}^{f^{\bar{u}_{2}}}$. And since $\tilde{u}_{1}$ satisfies (a) above, strategy-proofness of $f^{\bar{u}_{2}}$ implies $f_{1}^{\bar{u}_{2}}\left(\tilde{u}_{1}\right)=f_{1}\left(\tilde{u}_{1}, \bar{u}_{2}\right)=\left(\bar{x}, y_{1}\right)$ for some $y_{1} \geq \bar{y}_{1}$. Hence

$$
\hat{u}_{2}\left(f_{2}\left(\tilde{u}_{1}, \hat{u}_{2}\right)\right)=\hat{v}(\overline{\bar{x}})<\hat{v}(\bar{x})=\hat{u}_{2}\left(f_{2}\left(\tilde{u}_{1}, \bar{u}_{2}\right)\right),
$$


and therefore $f$ is manipulable at $\left(\tilde{u}_{1}, \hat{u}_{2}\right)$, which contradicts that $f$ is strategy-proof. (The functions $\tilde{u}_{1}$ and/or $\hat{u}_{2}$ used in this argument may not be concave. Note, however, that under the assumptions of Theorem 1 the sets $U_{1}$ and $U_{2}$ contain all the continuous utility functions, concave or not concave.)

Finally, we complete the proof of Lemma 3.2 under the assumptions of Theorem 2. Let $\left\{\hat{u}_{2}^{(k)}\right\}$ be a sequence of utility functions given for each integer $k$ by

$$
\hat{u}_{2}^{(k)}\left(x, y_{2}\right)=\hat{v}^{(k)}(x)=-(x-\overline{\bar{x}})^{\top} H^{(k)}(x-\overline{\bar{x}}),
$$

where $H^{(k)}$ a positive definite matrix chosen so that the level curves of $\hat{v}^{(k)}$ are elliptical balls obtained by shrinking a standard ball by a factor of $1 / k$ in all directions orthogonal to the line segment $[\bar{x}, \overline{\bar{x}}]$. (Zhou (1990) uses this construction in the proof of his Theorem 2, Step 3.) Since each $\hat{v}^{(k)}$ is uniquely maximized at $\overline{\bar{x}}$, we have $f_{x}\left(\hat{u}_{1}, \hat{u}_{2}^{(k)}\right)=f_{x}^{\hat{u}_{2}^{(k)}}\left(\hat{u}_{1}\right)=\overline{\bar{x}}$ for all $k$. For each $k$ write

$$
\omega^{(k)}=\max _{y_{1} \in Z_{Y_{1}}^{f^{(k)}}(k)} w_{(\bar{x})} w_{\bar{y}_{1}}\left(y_{1}\right) .
$$

Note $\omega^{(k)}$ is well defined by Lemma 3.1, and $\left\{\omega^{(k)}\right\} \subset[0,1]$ by the definition of the function $w_{\bar{y}_{1}}$. Let $\bar{\omega}$ be the limit of a subsequence of $\left\{\omega^{(k)}\right\}$. Henceforth we restrict attention to this subsequence, and to reduce notation we denote it also by $\left\{\omega^{(k)}\right\}$. We show that $\bar{\omega}<1$. Assume that $\bar{\omega}=1$; then for sufficiently large $k$ the set $Z_{Y_{1}}^{f_{u_{2}}^{\hat{u}^{(k)}}}(\bar{x})$ must contain points $y_{1}$ arbitrarily closed to the set $\left\{y_{1} \in Y_{1} \mid y_{1} \geq \bar{y}_{1}\right\}$. Since $\hat{u}_{1}$ satisfies (a) above, this in turn implies that for $k$ sufficiently large there is $\left(\bar{x}, y_{1}^{(k)}\right) \in Z_{1}^{f^{\hat{u}_{2}^{(k)}}}$ such that $\hat{u}_{1}\left(\bar{x}, y_{1}^{(k)}\right)>\hat{u}_{1}\left(\overline{\bar{x}}, y_{1}\right)$ for all $\left(\overline{\bar{x}}, y_{1}\right) \in Z_{1}^{f}$, and therefore $f_{x}^{\hat{u}_{2}^{(k)}}\left(\hat{u}_{1}\right)=\overline{\bar{x}}$ contradicts that $f^{\hat{u}_{2}^{(k)}}$ maximizes $\hat{u}_{1}$ (i.e., $f^{\hat{u}_{2}^{(k)}}$ is not strategy-proof). Since $\bar{\omega}<1$, then for $\delta \in(0,1-\bar{\omega})$ sufficiently small, there is no $k$ and $\left(x, y_{1}\right) \in Z_{1}^{f^{\hat{u}_{2}(k)}}$ such that $\|x-\bar{x}\|<\delta$ and $w_{\bar{y}_{1}}\left(y_{1}\right)>1-\delta$.

Let $\left(\overline{\bar{x}}, \overline{\bar{y}}_{1}\right) \in Z_{1}^{f}$ be such that $\bar{x}, \overline{\bar{x}}$ and $\overline{\bar{x}}$ are in general position. Choose $\delta<$ $\|\overline{\bar{x}}-\bar{x}\|$, and let $\varepsilon>0$ be sufficiently small that if $x \in X$ satisfies $\|x-\bar{x}\|>\delta$ and $d(x,[\bar{x}, \overline{\bar{x}}])<\varepsilon$, then $d(x,[\bar{x}, \overline{\bar{x}}])>\varepsilon$. Also let $\bar{k}$ be sufficiently large that $\hat{v}^{(\bar{k})}(\bar{x})>$ $\hat{v}^{(\bar{k})}(x)$ for all $x \in X$ such that $d(x,[\bar{x}, \overline{\bar{x}}])>\varepsilon$. Finally, let $\tilde{u}_{1} \in U_{1}$ be a concave utility function satisfying (a) and such that

(c.2) $\tilde{u}_{1}\left(\overline{\bar{x}}_{,}, \overline{\bar{y}}_{1}\right)>\tilde{u}_{1}\left(x, y_{1}\right)$ for all $\left(x, y_{1}\right) \in Z_{1}^{f^{\hat{u}_{2}^{(\bar{k})}}}$ such that $d(x,[\bar{x}, \overline{\bar{x}}])<\varepsilon$.

See Figure 1 ; in this figure the ellipse around the line segment $[\bar{x}, \overline{\bar{x}}]$ is the projection on $X$ of the indifference curve $\tilde{u}_{1}\left(x, y_{1}\right)=\tilde{u}_{1}(\overline{\bar{x}}, 0)$. (A utility function with these properties is constructed in Appendix C.) Since $f^{\hat{u}_{2}^{(\bar{k})}}$ is strategy-proof, then 
$f_{x}^{\hat{u}_{2}^{(\bar{k})}}\left(\tilde{u}_{1}\right)=\hat{x}^{(\bar{k})}$ satisfies $d\left(\hat{x}^{(\bar{k})},[\bar{x}, \overline{\bar{x}}]\right)>\varepsilon$. Hence

$$
\hat{u}_{2}^{(\bar{k})}\left(f_{2}\left(\tilde{u}_{1}, \hat{u}_{2}\right)\right)=\hat{v}^{(\bar{k})}\left(f_{x}^{\hat{u}_{2}^{(\bar{k})}}\left(\tilde{u}_{1}\right)\right)=\hat{v}^{(\bar{k})}\left(\hat{x}^{(k)}\right)<\hat{v}^{(\bar{k})}(\bar{x}) .
$$

Now, since $\left(\bar{x}, \bar{y}_{1}\right) \in Z_{1}^{f}$, then there is $\bar{u}_{2} \in U_{2}$ such that $\left(\bar{x}, \bar{y}_{1}\right) \in Z_{1}^{f^{\bar{u}_{2}}}$. And since $\tilde{u}_{1}$ satisfies (a) above, then strategy-proofness of $f^{\bar{u}_{2}}$ implies $f_{1}^{\bar{u}_{2}}\left(\tilde{u}_{1}\right)=f_{1}\left(\tilde{u}_{1}, \bar{u}_{2}\right)=$ $\left(\bar{x}, y_{1}\right)$ with $y_{1} \geq \bar{y}_{1}$. Therefore

$$
\hat{u}_{2}^{(\bar{k})}\left(f_{2}\left(\tilde{u}_{1}, \hat{u}_{2}^{(\bar{k})}\right)\right)=\hat{v}^{(\bar{k})}\left(\hat{x}^{(k)}\right)<\hat{v}^{(\bar{k})}(\bar{x})=\hat{u}_{2}\left(f_{2}\left(\tilde{u}_{1}, \bar{u}_{2}\right)\right) .
$$

Hence $f$ is manipulable at $\left(\tilde{u}_{1}, \hat{u}_{2}^{(\bar{k})}\right)$, which contradicts that $f$ is strategy-proof.

We now establish theorems 1 and 2. The proof of both theorems is identical, and therefore in the proof we refer to them as "the theorem."

Proof of theorems 1 and 2. Let $f$ be a strategy-proof mechanism satisfying the assumptions of the theorem. We show by induction on the number of individuals that $f$ is dictatorial for $n \geq 2$. (For $n=1$ strategy-proofness and dictatorship are equivalent properties.)

Assume that $n=2$. By Lemma 1 the restriction of $f$ to $U^{x}$ is dictatorial. Assume, w.l.o.g., that individual 1 is the dictator for $f$ on $U^{x}$. We show that individual 1 is a dictator for $f$. Let $\left(u_{1}, u_{2}\right) \in U$ and let $\left(\bar{x}, \bar{y}_{1}\right) \in Z_{1}^{f}$ arbitrary. By Lemma 3.2 there is $y_{1}^{\prime} \in Z_{Y_{1}}^{f_{2}}(\bar{x})$ such that $y_{1}^{\prime} \geq \bar{y}_{1}$. Hence there is $u_{1}^{\prime} \in U_{1}$ such that $f_{1}^{u_{2}}\left(u_{1}^{\prime}\right)=\left(\bar{x}, y_{1}^{\prime}\right)$. Thus, we have

$$
\begin{aligned}
u_{1}\left(f_{1}\left(u_{1}, u_{2}\right)\right) & =u_{1}\left(f_{1}^{u_{2}}\left(u_{1}\right)\right) \\
\text { (since } \left.f^{u_{2}} \text { is strategy-proof by Lemma } 2.1\right) & \geq u_{1}\left(f_{1}^{u_{2}}\left(u_{1}^{\prime}\right)\right) \\
& =u_{1}\left(\bar{x}, y_{1}^{\prime}\right) \\
\left(\text { since } u_{1} \text { is } y_{1}\right. \text {-increasing) } & \geq u_{1}\left(\bar{x}, \bar{y}_{1}\right) .
\end{aligned}
$$

Hence individual 1 is a dictator for $f$.

Assume that $f$ is dictatorial whenever $n<k$ for some $k \geq 2$, and suppose that $n=k$. Again by Lemma 1 the restriction of $f$ to $U^{x}$ is dictatorial. Assume, w.l.o.g., that individual 1 is the dictator for $f$ on $U^{x}$. We show that individual 1 is a dictator for $f$. Note that for all $i \in\{2, \ldots, n\}$ and $u_{i} \in U_{i}$ the mechanism $f^{u_{i}}$ is strategyproof and satisfies $Z_{X}^{f_{i}^{u_{i}}}=Z_{X}^{f}$ by Lemma 2.1. Hence $f^{u_{i}}$ satisfies the assumptions of the theorem and involves $k-1$ individuals, and therefore is dictatorial by the induction hypothesis. Moreover, the dictator of $f^{u_{i}}$ is individual 1 by Lemma 2.2. Let 
$u=\left(u_{1}, \ldots, u_{n}\right) \in U$ and let $\left(\bar{x}, \bar{y}_{1}\right) \in Z_{1}^{f}$ arbitrary. Then there is $u^{\prime}=\left(u_{1}^{\prime}, \ldots, u_{n}^{\prime}\right) \in U$ such that $f_{1}\left(u^{\prime}\right)=\left(\bar{x}, \bar{y}_{1}\right)$. Thus, we have

$$
\begin{aligned}
u_{1}\left(f_{1}\left(u_{1}, \ldots, u_{n}\right)\right) & =u_{1}\left(f_{1}^{u_{2}}\left(u_{1}, u_{-\{1,2\}}\right)\right) \\
\text { (since individual } \left.1 \text { is a dictator for } f^{u_{2}}\right) & \geq u_{1}\left(f_{1}^{u_{2}}\left(u_{1}^{\prime}, u_{-\{1,2\}}^{\prime}\right)\right) \\
& =u_{1}\left(f_{1}^{u_{3}^{\prime}}\left(u_{1}^{\prime}, u_{2}, u_{-\{1,2,3\}}^{\prime}\right)\right) \\
\text { (since individual } \left.1 \text { is a dictator for } f^{u_{3}^{\prime}}\right) & =u_{1}\left(f_{1}^{u_{3}^{\prime}}\left(u_{1}^{\prime}, u_{2}^{\prime}, u_{-\{1,2,3\}}^{\prime}\right)\right) \\
& =u_{1}\left(f_{1}\left(u^{\prime}\right)\right) \\
& =u_{1}\left(\bar{x}_{1}, \bar{y}_{1}\right) .
\end{aligned}
$$

Therefore individual 1 is a dictator for $f$, thereby establishing the theorem.

Proof of Theorem 3. Let $f$ be a strategy-proof and efficient mechanism. We show $Z_{X} \subset Z_{X}^{f}$, and therefore that $Z_{X}^{f}=Z_{X}$. Let $(\bar{x}, \bar{y}) \in Z$ arbitrary, and for $i \in N$ let $\bar{u}_{i}\left(x, y_{i}\right)=-\|x-\bar{x}\|$. If $f_{x}\left(\bar{u}_{1}, \ldots, \bar{u}_{n}\right) \neq \bar{x}$, then $(\bar{x}, \bar{y})$ is Pareto superior to $f\left(\bar{u}_{1}, \ldots, \bar{u}_{n}\right)$ contradicting that $f$ is efficient. Hence $f_{x}\left(\bar{u}_{1}, \ldots, \bar{u}_{n}\right)=\bar{x}$ and $\bar{x} \in Z_{X}^{f}$. Thus, under (3.1) $f$ satisfies the assumptions of Theorem 1, and under (3.2) $f$ satisfies the assumptions of Theorem 2, and therefore $f$ is dictatorial. Assume w.l.o.g. that individual 1 is the dictator. We show that he is a strong dictator.

Assume by way of contradiction that there are $u \in U$ and $(\bar{x}, \bar{y}) \in Z$ such that $u_{1}\left(\bar{x}, \bar{y}_{1}\right)>u_{1}\left(f_{1}(u)\right)$. Since $f_{1}(u)$ maximizes $u_{1}$ on $Z_{1}^{f}$, we have $u_{1}\left(\bar{x}, \bar{y}_{1}\right)>u_{1}\left(x, y_{1}\right)$ for all $\left(x, y_{1}\right) \in Z_{1}^{f}$. For $i \in N$, let $\bar{u}_{i}\left(x, y_{i}\right)=-\|x-\bar{x}\|$. Then

$$
\bar{u}_{i}\left(\bar{x}, \bar{y}_{i}\right) \geq \bar{u}_{i}\left(f_{i}\left(u_{1}, \bar{u}_{2}, \ldots, \bar{u}_{n}\right)\right)
$$

for all $i \in N \backslash\{1\}$, and

$$
u_{1}\left(\bar{x}, \bar{y}_{1}\right)>u_{1}\left(f_{1}\left(u_{1}, \bar{u}_{2}, \ldots, \bar{u}_{n}\right)\right)
$$

i.e., $(\bar{x}, \bar{y})$ is Pareto superior to $f\left(u_{1}, \bar{u}_{2}, \ldots, \bar{u}_{n}\right)$ at $\left(u_{1}, \bar{u}_{2}, \ldots, \bar{u}_{n}\right)$, which contradicts that $f$ is an efficient mechanism.

\section{Appendix A}

We construct a utility function satisfying the properties (a) and (b) specified in the proof of Lemma 3.2. Figures $2 \mathrm{a}$ and $2 \mathrm{~b}$ illustrate this construction for the cases $X=\mathbb{R}_{+}$and $X=\mathbb{R}_{+}^{2}$, respectively. 
We first construct a real-valued function $g$ on $X \times \mathbb{R}_{+}$. Let $\overline{\bar{x}} \in Z_{X}^{f} \backslash\{\bar{x}\}$. Denote by $R_{0}$ the vertical line in $X \times \mathbb{R}_{+}$passing $(\overline{\bar{x}}, 0)$, i.e., the line containing all the points $(\overline{\bar{x}}, \omega)$ for $\omega \in \mathbb{R}_{+}$, and denote by $R_{1}$ the line passing $(\overline{\bar{x}}, 0)$ and $(\bar{x}, \hat{\omega})$, where

$$
\hat{\omega}=\frac{1}{2}\left(1+\max _{y_{1} \in Z_{Y_{1}}^{f^{u_{2}}}(\bar{x})} w_{\bar{y}_{1}}\left(y_{1}\right)\right) .
$$

Note that $\hat{\omega}$ is well defined by Lemma 3.1. Moreover, $\bar{y}_{1} \notin Z_{Y_{1}}^{f_{2}}(\bar{x})$ implies

$$
0 \leq \max _{y_{1} \in Z_{Y_{1}}^{f_{2}}(\bar{x})} w_{\bar{y}_{1}}\left(y_{1}\right)<\hat{\omega}<1
$$

Denote by $\alpha \in(0, \pi / 2)$ the angle formed by $R_{0}$ and $R_{1}$; i.e., $\alpha$ is defined by the equation

$$
\tan \alpha=(1 / \hat{\omega}) \sqrt{(\bar{x}-\overline{\bar{x}})^{\top}(\bar{x}-\overline{\bar{x}})} .
$$

Also, let $R_{2}$ be the half line passing $(\overline{\bar{x}}, 0)$ and $\left(\bar{x}, w_{\bar{y}_{1}}\left(\bar{y}_{1}\right)\right)=(\bar{x}, 1)$ containing the points $(x, \omega) \in X \times \mathbb{R}_{+}$given by $(t \overline{\bar{x}}+(1-t) \bar{x},(1-t))$ for $t \geq 0$. For $t \geq 0$ denote by $K(t)$ the cone with angle $\alpha$ and vertex in the point on $R_{2}$ defined by $t$; i.e., $K(t)$ contains the points $(x, \omega)$ such that

$$
\omega-(1-t)=(1 / \tan \alpha) \sqrt{(x-(t \overline{\bar{x}}+(1-t) \bar{x}))^{\top}(x-(t \overline{\bar{x}}+(1-t) \bar{x}))} .
$$

Also denote by $C(t)$ the cylinder containing the of points $(x, \omega)$ such that

$$
t=(1 / \tan \alpha) \sqrt{(x-(t \overline{\bar{x}}+(1-t) \bar{x}))^{\top}(x-(t \overline{\bar{x}}+(1-t) \bar{x}))} .
$$

Note that for each $(x, \omega) \in X \times \mathbb{R}_{+}$there is a unique line $R$ passing $(x, \omega)$ and forming an angle $\alpha \in(0, \pi / 2)$ with a vertical line $R_{0}$; the intersection of $R$ with the half line $R_{2}$ identifies the unique cone that contains $(x, \omega)$, which is identified by the unique solution $t \geq 0$ to the equation defining $K(t)$ above. Likewise each point $(x, \omega) \in X \times \mathbb{R}_{+}$is contained in a unique cylinder, which is identified by the unique solution $t \geq 0$ to the equation defining $C(t)$ above.

Let the function $g: X \times \mathbb{R}_{+} \rightarrow \mathbb{R}$ be given for $(x, \omega) \in X \times \mathbb{R}_{+}$by $g(x, \omega)=-t$, where $t \geq 0$ identifies the cone $K(t)$ that contains $(x, \omega)$ if $\omega<1$, and $t$ identifies the cylinder $C(t)$ that contains $(x, \omega)$ if $\omega \geq 1$. It is easy to see that $g$ is continuous: for $(x, \omega) \in X \times \mathbb{R}_{+}$such that $\omega<1$ (respectively, $\omega>1$ ) the equation identifying $K(t)$ (respectively, the equation identifying $C(t)$ ) that defines implicitly the value of $g$ ( $t$ in these equations) involves continuous functions; further, as $\omega$ approaches 1 the equation defining $K(t)$ becomes that defining $C(t)$. Moreover, $g$ is concave as each 
of its level curves $g(x, \omega)=-t$ is formed by the union of the cone $K(t)$ truncated horizontally above $\omega=1$ and the cylinder $C(t)$ truncated horizontally below $\omega=1$. (See figures $2 \mathrm{a}$ and $2 \mathrm{~b}$. )

Let the function $\hat{u}_{1}: X \times Y_{1} \rightarrow \mathbb{R}$ be given for $\left(x, y_{1}\right) \in X \times Y_{1}$ by $\hat{u}_{1}\left(x, y_{1}\right)=$ $g\left(x, w_{\bar{y}_{1}}\left(y_{1}\right)\right)$. The function $\hat{u}_{1}$ is continuous as it is the composition of continuous functions. We show that $\hat{u}_{1}$ is $y_{1}$-increasing. Let $\left(x, y_{1}\right),\left(x, y_{1}^{\prime}\right) \in X \times Y_{1}$ with $y_{1}>y_{1}^{\prime}$; then $w_{\bar{y}_{1}}\left(y_{1}\right) \geq w_{\bar{y}_{1}}\left(y_{1}^{\prime}\right)$. Write $\hat{u}_{1}\left(x, y_{1}\right)=-t, \hat{u}_{1}\left(x, y_{1}^{\prime}\right)=-t^{\prime}$. If $w_{\bar{y}_{1}}\left(y_{1}\right)=w_{\bar{y}_{1}}\left(y_{1}^{\prime}\right)=$ 1 , then $\left(x, w_{\bar{y}_{1}}\left(y_{1}\right)\right)=\left(x, w_{\bar{y}_{1}}\left(y_{1}^{\prime}\right)\right)$, and therefore $t=t^{\prime}$. If $w_{\bar{y}_{1}}\left(y_{1}\right)=1>w_{\bar{y}_{1}}\left(y_{1}^{\prime}\right)$, then

$$
t=w_{\bar{y}_{1}}\left(y_{1}^{\prime}\right)-\left(1-t^{\prime}\right)=t^{\prime}-\left(1-w_{\bar{y}_{1}}\left(y_{1}^{\prime}\right)\right)<t^{\prime} .
$$

If $w_{\bar{y}_{1}}\left(y_{1}\right)<1$, then

$$
w_{\bar{y}_{1}}\left(y_{1}\right)-(1-t)=w_{\bar{y}_{1}}\left(y_{1}^{\prime}\right)-\left(1-t^{\prime}\right)
$$

i.e.,

$$
0 \geq w_{\bar{y}_{1}}\left(y_{1}\right)-w_{\bar{y}_{1}}\left(y_{1}^{\prime}\right)=t^{\prime}-t .
$$

We show that $\hat{u}_{1}$ is concave: For $\left(x, y_{1}\right),\left(x^{\prime}, y_{1}^{\prime}\right) \in X \times Y_{1}$ and $\lambda \in[0,1]$ we have

$$
\hat{u}_{1}\left(\lambda x+(1-\lambda) x^{\prime}, \lambda y_{1}+(1-\lambda) y_{1}^{\prime}\right)=g\left(\lambda x+(1-\lambda) x^{\prime}, w_{\bar{y}_{1}}\left(\lambda y_{1}+(1-\lambda) y_{1}^{\prime}\right)\right)
$$

(since $w_{\bar{y}_{1}}$ is concave, $g$ is $y_{1}$-increasing) $\geq g\left(\lambda x+(1-\lambda) x^{\prime}, \lambda w_{\bar{y}_{1}}\left(y_{1}\right)+(1-\lambda) w_{\bar{y}_{1}}\left(y_{1}^{\prime}\right)\right)$

$$
\begin{aligned}
\text { (since } g \text { is concave) } & \geq \lambda g\left(x, w_{\bar{y}_{1}}\left(y_{1}\right)\right)+(1-\lambda) g\left(x^{\prime}, w_{\bar{y}_{1}}\left(y_{1}^{\prime}\right)\right) \\
& =\lambda \hat{u}_{1}\left(x, y_{1}\right)+(1-\lambda) \hat{u}_{1}\left(x^{\prime}, y_{1}^{\prime}\right) .
\end{aligned}
$$

Now, since $\left(\bar{x}, w_{\bar{y}_{1}}\left(\bar{y}_{1}\right)\right)=(\bar{x}, 1) \in C(0)$, we have $\hat{u}_{1}\left(\bar{x}, \bar{y}_{1}\right)=0 \geq \hat{u}_{1}\left(x, y_{1}\right)$ for all $\left(x, y_{1}\right) \in X \times Y_{1} \supset Z_{1}^{f} \backslash\left\{\left(\bar{x}, y_{1}^{\prime}\right) \in Z_{1} \mid y_{1}^{\prime} \geq \bar{y}_{1}\right\}$. Hence $\hat{u}_{1}$ satisfies condition (a) of Lemma 3.2. Since $(\overline{\bar{x}}, 0),(\bar{x}, \hat{\omega}) \in K(1)$, and $w_{\bar{y}_{1}}\left(y_{1}\right)<\hat{\omega}$ for all $y_{1} \in Z_{Y_{1}}^{f^{u_{2}}}(\bar{x})$, then

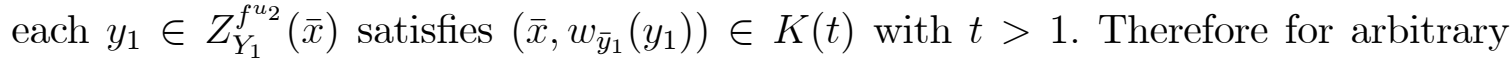

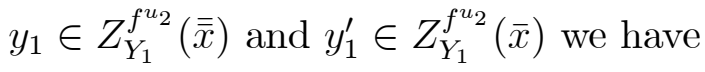

$$
\hat{u}_{1}\left(\overline{\bar{x}}, y_{1}\right) \geq \hat{u}_{1}(\overline{\bar{x}}, 0)=-1>\hat{u}_{1}\left(\bar{x}, y_{1}^{\prime}\right) \text {. }
$$

Hence if $(x, y)$ maximizes $\hat{u}_{1}$ on $Z_{Y_{1}}^{f^{u_{2}}}(\bar{x})$, we have $x \neq \bar{x}$; i.e., $\hat{u}_{1}$ satisfies condition (b) if Lemma 3.2.

\section{Appendix B}

We construct a utility function $\tilde{u}_{1} \in U_{1}$ with properties (a) and (c.1) specified in the proof of Lemma 3.2 under the assumptions of Theorem 1. This construction builds on a function used by Barberà and Peleg (1990) in the proof of their Lemma 5.6. 
Note that since there is no $y_{1} \in Z_{1}^{f^{\hat{u}_{2}}}(\bar{x})$ with $y_{1} \geq \bar{y}_{1}$, then

$$
\hat{u}_{1}\left(f_{1}\left(\hat{u}_{1}, \hat{u}_{2}\right)\right)=\max _{Z_{1}^{f_{2}}} \hat{u}_{1}\left(x, y_{1}\right)<\hat{u}_{1}\left(\bar{x}, \bar{y}_{1}\right) .
$$

Let $\delta>0$ sufficiently small that

$$
B:=\{x \in X \mid\|x-\bar{x}\|<\delta\} \times\left\{y_{1} \in Y_{1} \mid w_{\bar{y}_{1}}\left(y_{1}\right)>1-\delta\right\}
$$

is contained in $\left(Z_{1}^{f^{\hat{u}_{2}}}\right)^{C}$, and

$$
B_{\delta}(\overline{\bar{x}}):=\{x \in X \mid\|x-\overline{\bar{x}}\|<\delta\}
$$

satisfies $B_{\delta}(\overline{\bar{x}}) \cap B_{X}=\varnothing$. Define $\breve{u}_{1}: X \times Y_{1} \rightarrow \mathbb{R}$ for $\left(x, y_{1}\right) \in X \times Y_{1}$ by

$$
\breve{u}_{1}\left(x, y_{1}\right)=\frac{d\left(x, X \backslash B_{X}\right)}{\|x-\bar{x}\|+d\left(x, X \backslash B_{X}\right)} \breve{w}\left(y_{1}\right)
$$

if $\left(x, y_{1}\right) \in B$, and by $\breve{u}_{1}\left(x, y_{1}\right)=0$ if $\left(x, y_{1}\right) \notin B$, where

$$
\breve{w}\left(y_{1}\right)=\max \left\{0,\left(w_{\bar{y}_{1}}\left(y_{1}\right)-(1-\delta)\right) / \delta\right\}
$$

Note that $\breve{u}_{1}$ is is continuous on $X \times Y_{1}$ since it is zero on the boundary of $B$. Also define

$$
\breve{v}(x)=\frac{d\left(x, X \backslash B_{\delta}(\overline{\bar{x}})\right)}{2\left(\|x-\overline{\bar{x}}\|+d\left(x, X \backslash B_{\delta}(\overline{\bar{x}})\right)\right)}
$$

We check that the utility function $\tilde{u}_{1}\left(x, y_{1}\right)=\breve{u}_{1}\left(x, y_{1}\right)+\breve{v}(x)$ has the desired properties. Since $B_{\delta}(\overline{\bar{x}}) \cap B_{X}=\varnothing$, we have $\tilde{u}_{1}\left(x, y_{1}\right) \leq 1$ on $X \times Y_{1}$. Also $\tilde{u}_{1}\left(x, y_{1}\right)<1$ if either $x \neq \bar{x}$ or $y_{1} \nsucceq \bar{y}_{1}$ (i.e., $w_{\bar{y}_{1}}\left(y_{1}\right)<1$ ). Moreover $\tilde{u}_{1}\left(\bar{x}, y_{1}\right)=1$ for $y_{1} \geq \bar{y}_{1}$. Hence $\tilde{u}_{1}$ satisfies condition (a) of Lemma 3.2. Also since $B \subset\left(Z_{1}^{f^{\hat{u}_{2}}}\right)^{C}$, then $\tilde{u}_{1}\left(x, y_{1}\right)=$ $\breve{v}(x)$ on $Z_{1}^{f^{\hat{u}_{2}}}$, and $\breve{v}(x)<\breve{v}(\overline{\bar{x}})=1 / 2$ for all $x \in Z_{X}^{f^{\hat{u}_{2}}} \backslash\{\overline{\bar{x}}\}$. Thus, if $\left(x, y_{1}\right)$ maximizes $\tilde{u}_{1}$ on $Z_{1}^{f^{\hat{u}_{2}}}$, then $x=\overline{\bar{x}}$; i.e., $\tilde{u}_{1}$ satisfies (c.1) of Lemma 3.2 .

\section{Appendix $\mathrm{C}$}

We construct a utility function $\tilde{u}_{1} \in U_{1}$ with properties (a) and (c.2) specified in the proof of Lemma 3.2 under the assumptions of Theorem 2. This construction builds on the proof of Zhou (1990)'s Theorem 2, Step 3. The construction of $\tilde{u}_{1}$ is analogous to that the function $\hat{u}_{1}$ in Appendix A, except that $\overline{\bar{x}}$ plays the role of $\overline{\bar{x}}$, and we modify the construction of the function $g$ so that its indifference curves are elliptical cones rather than spherical cones. 
Denote by $\tilde{R}_{0}$ the vertical line on $X \times \mathbb{R}_{+}$passing $(\overline{\bar{x}}, 0)$, and let $\tilde{R}_{1}$ the line passing $(\overline{\bar{x}}, 0)$ and $(\bar{x}, \tilde{\omega})$, where $\tilde{\omega}=\frac{1}{2}(1+\bar{\omega})$. (Here $\bar{\omega}$ is the subsequential limit used in the argument of Lemma 3.2.) Also, let $\tilde{R}_{2}$ be the half line passing $(\overline{\bar{x}}, 0)$ and $(\bar{x}, 1)$ containing the points $(t \overline{\bar{x}}+(1-t) \bar{x},(1-t))$ for $t \geq 0$. For $t \geq 0$ denote by $\tilde{K}(t)$ the elliptical cone with vertex in the point on $\tilde{R}_{2}$ defined by $t$, containing the points $(x, \omega)$ such that

$$
\omega-(1-t)=D \sqrt{(x-(t \overline{\bar{x}}+(1-t) \bar{x}))^{\top} H(x-(t \overline{\bar{x}}+(1-t) \bar{x}))},
$$

where $D$ is a constant satisfying $0<D<\tilde{\omega} /\|\bar{x}-\overline{\bar{x}}\|$, and $H$ is a positive definite matrix, which are chosen in such a way that for fixed $\omega$ the points $(x, \omega) \in \tilde{K}(1)$ form an elliptical ball obtained by shrinking a spherical ball in all directions orthogonal to the line $\tilde{R}_{1}$, so that for $\|x-\bar{x}\|>\delta$ we have $d(x,[\bar{x}, \overline{\bar{x}}])>\varepsilon-$ see Figure 1 . Also denote by $\tilde{C}(t)$ the elliptical cylinder containing the points $(x, \omega)$ such that

$$
t=D \sqrt{(x-(t \overline{\bar{x}}+(1-t) \bar{x}))^{\top} H(x-(t \overline{\bar{x}}+(1-t) \bar{x}))} .
$$

Let $\tilde{g}: X \times \mathbb{R}_{+} \rightarrow \mathbb{R}$ be given for $(x, \omega) \in X \times \mathbb{R}_{+}$by $\tilde{g}(x, \omega)=-t$, where $t \geq 0$ identifies the cone $\tilde{K}(t)$ that contains $(x, \omega)$ if $\omega<1$, and $t$ identifies the cylinder $\tilde{C}(t)$ that contains $(x, \omega)$ if $\omega \geq 1$. Finally, let $\tilde{u}_{1}: X \times Y_{1} \rightarrow \mathbb{R}$ be given for $\left(x, y_{1}\right) \in X \times Y_{1}$ by $\tilde{u}_{1}\left(x, y_{1}\right)=\tilde{g}\left(x, w_{\bar{y}_{1}}\left(y_{1}\right)\right)$.

Since $\left(\bar{x}, w_{\bar{y}_{1}}\left(\bar{y}_{1}\right)\right)=(\bar{x}, 1) \in \tilde{C}(0)$, then as shown in Appendix A the function $\tilde{u}_{1}$ satisfies condition $(\mathrm{a})$. And since $\left(\overline{\bar{x}}, w_{\bar{y}_{1}}(0)\right)=(\overline{\bar{x}}, 0) \in \tilde{K}(1)$, and by construction every point $(x, \omega)$ inside the elliptical cone $\tilde{K}(1)$ satisfies $d(x,[\bar{x}, \overline{\bar{x}}])>\varepsilon$, then for $\left(x, y_{1}\right) \in X \times Y_{1}$ such that

$$
\tilde{u}_{1}\left(x, y_{1}\right)=\tilde{g}\left(x, w_{\bar{y}_{1}}\left(y_{1}\right)\right)>\tilde{g}(\overline{\bar{x}}, 0)=\tilde{u}_{1}(\overline{\bar{x}}, 0) \geq \tilde{u}_{1}\left(\overline{\bar{x}}, \overline{\bar{y}}_{1}\right),
$$

we have $d(x,[\bar{x}, \overline{\bar{x}}])>\varepsilon$. Therefore $\tilde{u}_{1}$ satisfies (c.2) of Lemma 3.2. 


\section{References}

[1] Barberà, S. and M. Jackson: Strategy-proof exchange. Econometrica 63, 51-87 (1995).

[2] Barberà, S. and B. Peleg: Strategy-proof voting schemes with continuous preferences. Social Choice and Welfare 7, 31-38 (1990).

[3] Barberà, S.: Strategy-Proof Social Choice. Barcelona GSE working paper num. 40 (2010).

[4] Bergemann, D. and S. Morris: Robust Implementation in Direct Mechanisms. Review of Economic Studies 76, 1175-1204 (2009).

[5] Deb, R. S. Ohseto: Strategy-proof and Individually Rational Social Choice Functions for Public Good Economies: A Note. Economic Theory 14, 685-689 (1999).

[6] Gibbard, A.: Manipulation of voting schemes: a general result. Econometrica 41, 587-602 (1973).

[7] Green, J. and J.-J. Laffont: Characterization of satisfactory mechanisms for the revelation of preferences for public goods. Econometrica 45, 427-438 (1977).

[8] Hashimoto, K.: Strategy-proofness versus Efficiency on the Cobb-Douglas Domain of Exchange Economies. Social Choice and Welfare, 31, 457-473 (2008).

[9] Hurwicz, L.: On informationally decentralized systems, in Decisions and Organization. Ed. by E. McGuire and R. Radner. Amsterdam, North-Holland, 297-336 (1972).

[10] Hurwicz, L. and M. Walker: On the generic non-optimality of dominant-strategy allocation mechanisms: a general results that includes pure exchange economies. Econometrica 58, 683-704 (1990).

[11] Ju, B.-G.: Strategy-proofness versus Efficiency in Exchange Economies: General Domain Properties and Applications. Social Choice and Welfare 21, 73-93 (2003)

[12] Ledyard, J.: Incomplete information and incentive compatibility. Journal of Economic Theory 18, 171-189 (1978).

[13] Lindahl, E.: Just taxation: a positive solution. In Classics in the Theory of Public Finance. Ed. by R. Musgrave and A. Peacock . Macmillan (1964). 
[14] Moreno, D.: Nonmanipulable decision mechanisms for economic environments. Social Choice and Welfare 11, 225-240 (1994).

[15] Moreno, D.: Strategy-proof allocation mechanisms for pure public goods economies when preferences are monotonic. Economic Theory 13, 183-197 (1999).

[16] Moreno, D. and M. Walker: Nonmanipulable voting schemes when participant's interests are partially decomposable. Social Choice and Welfare 8, 221-233 (1991).

[17] Moulin, H.: On strategy-proofness and single peakedness. Public Choice 34, 87-97 (1980).

[18] Nicoló, A.: Efficiency and Truthfulness with Leontief Preferences. A Note on Two-Agent, Two-Good Economies. Review of Economic Design, 8, 373-382 (2004).

[19] Saijo, T.: Incentive Compatibility and Individual Rationality in Public Good Economies. Journal of Economic Theory 55, 203-212 (1991).

[20] Samuelson, P.: The pure theory of public expenditure. Review of Economics and Statistics 36, 387-389 (1954).

[21] Satterthwaite, M.: Strategy-proofness and Arrow's conditions: existence and correspondence theorems for voting procedures and social welfare functions. Journal of Economic Theory 35, 1-18 (1975).

[22] Satterthwaite, M. and H. Sonnenschein: Strategy-proof allocation mechanisms at differentiable points. Review of Economics Studies 48, 587-597 (1981).

[23] Schummer, J.: Strategy-proofness versus Efficiency on Restricted Domains of Exchange Economies. Social Choice and Welfare 14, 47-56 (1997).

[24] Schummer, J.: Strategy-proofness versus Efficiency for Small Domains of Preferences over Public Goods. Economic Theory 13, 709-722 (1999).

[25] Serizawa, S.: Strategy-Proof and Individually Rational Social Choice Functions for Public Goods Economies. Economic Theory 7, 501-512 (1996).

[26] Serizawa, S.: Strategy-Proof and Symmetric Social Choice Functions for Public Goods Economies. Econometrica 67, 121-145 (1999). 
[27] Serizawa, S.: Inefficiency of Strategy-Proof Rules for Pure Exchange Economies. Journal of Economic Theory 106, 219-241 (2002).

[28] Serizawa, S. and J. Weymark: Efficient Strategy-Proof Exchange and Minimum Consumption Guarantees. Journal of Economic Theory 109, 246-263 (2003).

[29] Walker, M.: On the nonexistence of a dominant strategy mechanism for making optimal public decision. Econometrica 48, 1521-1540 (1980).

[30] Wilson, R.: Game Theoretic Analysis of Trading Processes. In Advances in Economic Theory. Ed. by T. Bewley, Cambridge University Press (1987).

[31] Zhou, L.: Impossibility of strategy-proof allocation mechanisms in economies with public goods. Review of Economics Studies 58,107-119 (1991).

[32] Zhou, L: Inefficiency of strategy-proof allocation mechanisms in pure exchange economies. Social Choice and Welfare 8, 247-254 (1991). 


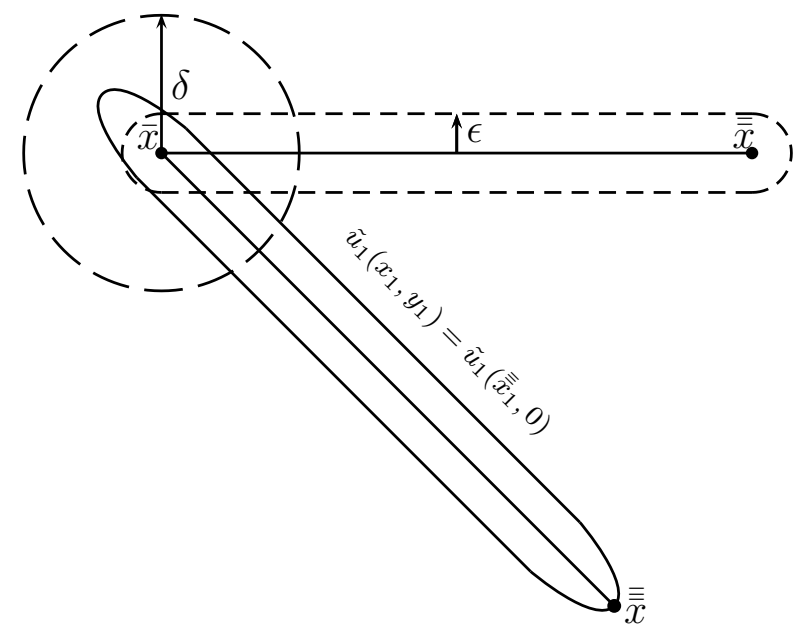

Figure 1 


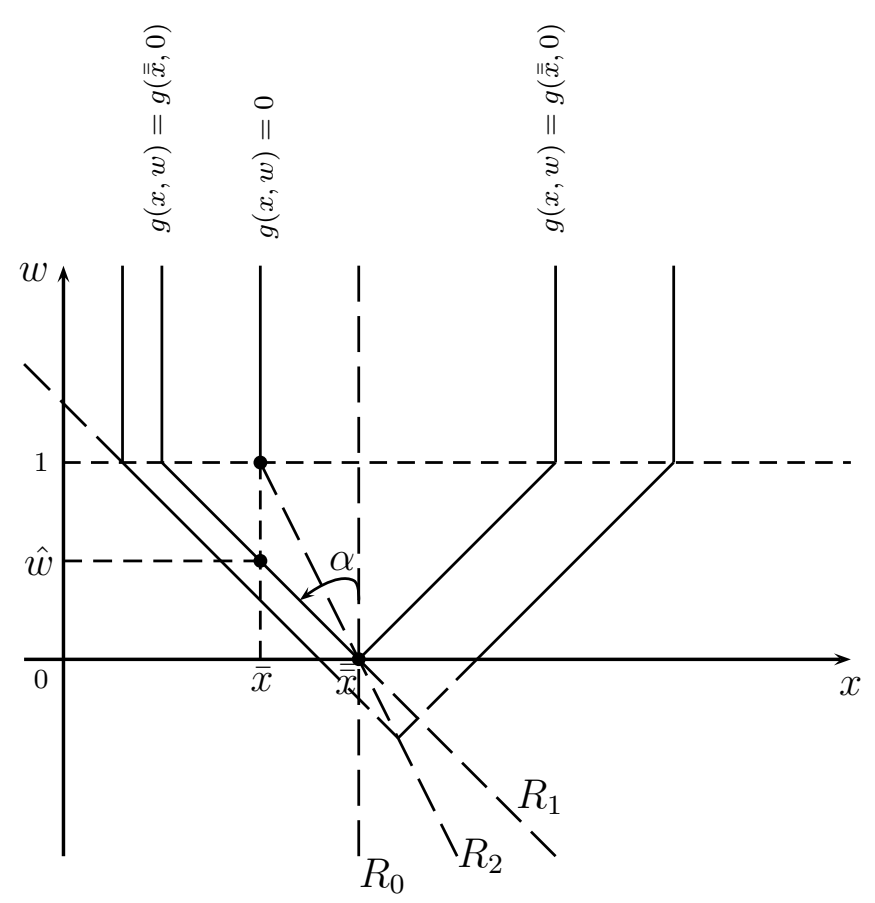

Figure 2a: $X=\mathbb{R}_{+}$ 


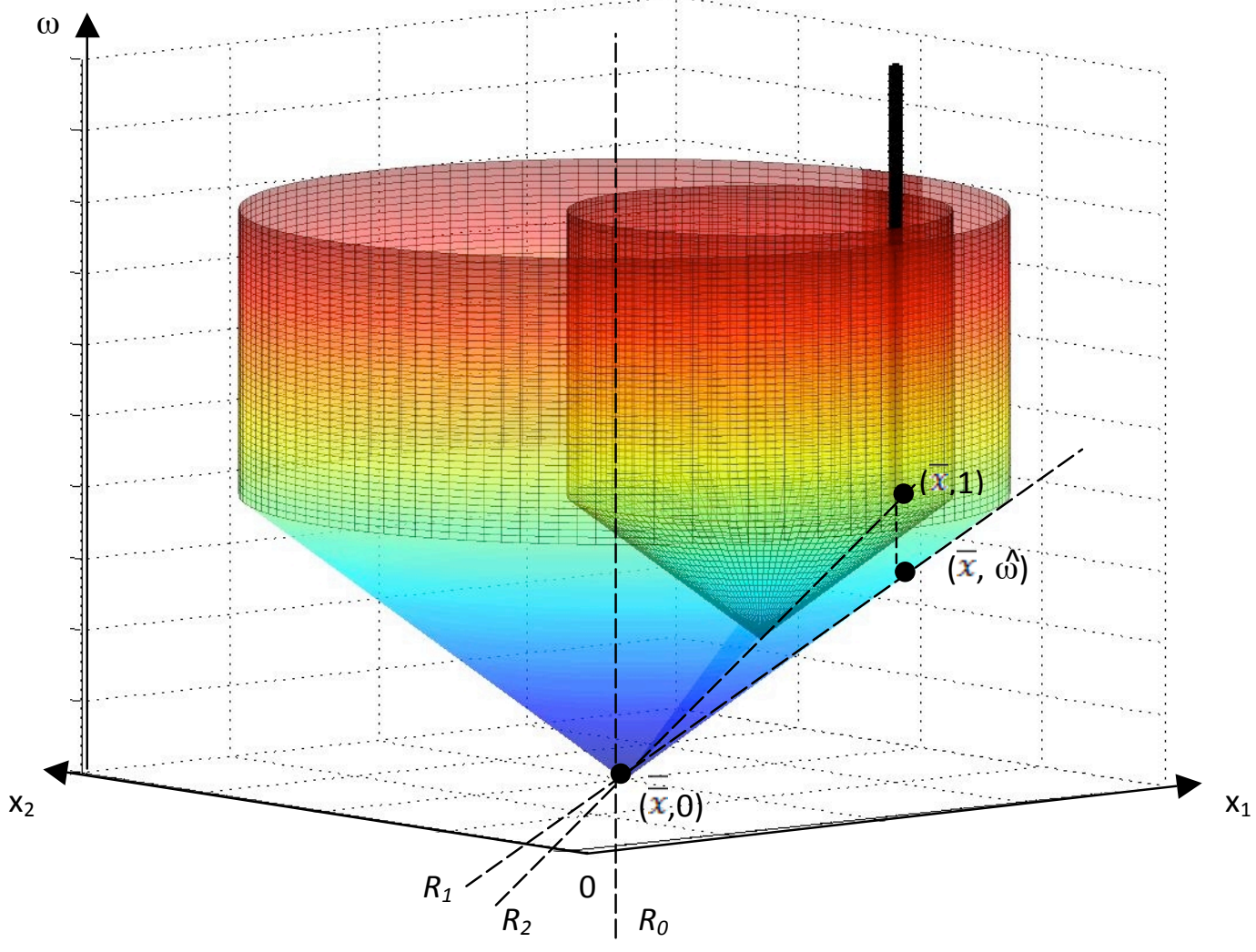

Figure $2 b . X=\Re_{+}{ }^{2}$ 